\title{
Spectroscopy and exotica of heavy flavor states in ATLAS
}

\section{Radek Novotny on behalf of the ATLAS collaboration*}

FNSPE CTU in Prague, Brehova 7,Prague, Czech Republic

E-mail: radek. novotny@cern.ch

\begin{abstract}
Several studies were performed using $4.9 \mathrm{fb}^{-1}$ of $\sqrt{s}=7 \mathrm{TeV}$ and $19.2 \mathrm{fb}^{-1}$ of $\sqrt{s}=8 \mathrm{TeV} p p$ collision data collected with the ATLAS detector at the LHC and the results of the latest studies are presented here. The first study is devoted to the search for a structure in the $B_{s}^{0} \pi^{ \pm}$invariant mass spectrum in the ATLAS detector particularly the search for the resonance $X(5568)$ which is a tetra-quark candidate. The second study is devoted to the search for excited states of the $B_{c}^{ \pm}$ through its hadronic transition to the ground state, detected in the decay $B_{c}^{ \pm} \rightarrow J / \psi \pi^{ \pm}$. The state appears in the $m\left(B_{c}^{ \pm} \pi^{+} \pi^{-}\right)-m\left(B_{c}^{ \pm}\right)-2 m\left(\pi^{ \pm}\right)$mass difference distribution with a significance of 5.2 standard deviations.
\end{abstract}

XIV International Conference on Heavy Quarks and Leptons (HQL2018)

May 27- June 1, 2018

Yamagata Terrsa, Yamagata,Japan

${ }^{*}$ Speaker. 


\section{Search for the state $X(5568) \rightarrow B_{s}^{0} \pi^{ \pm}$}

\subsection{Introduction}

In early 2016, the D $\emptyset$ collaboration [1] announced the evidence of a narrow structure referred to as $\mathrm{X}(5568)$, in $B_{s}^{0} \pi^{ \pm}$spectrum in $p \bar{p}$ collisions at $\sqrt{s}=1.96 \mathrm{TeV}$ center-of-mass energy. The most probable explanation of such as an exotic state would be a hadron composed of four quarks $(b, s, u, d)$. This exotic state would be very important for understanding of the production mechanism of multiquark objects and in the case of the tightly bound di-quark anti-diquark pair, this may provide an additional information to strong interaction potential.

The D $\emptyset$ collaboration reported the signal with $5.1 \sigma$ significance with invariant mass $m=$ $5567.8 \pm 2.9$ (stat $)_{-1.9}^{+0.9}$ (syst) $\mathrm{MeV} / \mathrm{c}^{2}$ and natural width $\Gamma=21.9 \pm 6.4(\text { stat })_{-2.5}^{+5.0}$ (syst) $\mathrm{MeV} / \mathrm{c}^{2}$. The ratio $\rho_{X}$ of the yield of $\mathrm{X}(5568)$ to the yield of the $B_{s}^{0}$ meson for a transverse momentum range $10<p_{\mathrm{T}}\left(B_{s}^{0}\right)<30 \mathrm{GeV}$ was measured to be $0.086 \pm 0.019$ (stat) \pm 0.014 (syst).

This result from the D $\emptyset$ collaboration was further investigated by the LHCb [2], CMS [3] and CDF [4] collaborations. As none of these experiments saw any evidence of the reported state $X(5568)$, each published an upper limit on $95 \% \mathrm{CL}$ of the relative production rate $\rho_{X}$. Recently, the $\mathrm{D} \emptyset$ collaboration published further evidence for the $\mathrm{X}(5568)$ resonance in the decay sequence $X \rightarrow B_{s}^{0} \pi^{ \pm}, B_{s}^{0} \rightarrow \mu^{\mp} v D_{s}^{ \pm}, D_{s}^{ \pm} \rightarrow \phi \pi^{ \pm}$[5], which is consistent with their previous measurement.

In parallel to these measurements, search for $X(5568) \rightarrow B_{s}^{0} \pi^{ \pm}$was performed with the ATLAS detector [6]. The results of the analysis are presented in following sections in more detail.

\subsection{Data and candidate selection}

The studies were made on a data sample recorded with the ATLAS detector [7] at Large Hadron Collider corresponding to the $4.9 \mathrm{fb}^{-1}$ of $p p$ collision data at $\sqrt{s}=7 \mathrm{TeV}$ and $19.5 \mathrm{fb}^{-1}$ at $\sqrt{s}=8 \mathrm{TeV}$. To study the detector response, to estimate backgrounds and to model systematic effects several Monte Carlo (MC) simulations were used. The grand sample of $12 \cdot 10^{6} B_{s}^{0} \rightarrow J / \psi \phi$ and $1 \cdot 10^{6}$ of $B_{s}^{0} \pi^{ \pm}$events were generated using Pythia 8.18 tuned with ATLAS data.

In order to select $B_{s}^{0} \pi^{ \pm}$candidate, events collected with di-muon triggers were used, which are based on $J / \psi \rightarrow \mu^{+} \mu^{-}$identification with $p_{\mathrm{T}}$ threshold of either 4 or $6 \mathrm{GeV}$ and at least one reconstructed primary vertex (PV) formed from at least six inner detector (ID) tracks. For the selection of $B_{s}^{0} \rightarrow J / \psi \phi$ candidates, the kaon tracks with $p_{\mathrm{T}}$ greater than $1 \mathrm{GeV}$ are required and only the best $\chi^{2}$ candidates with $\chi^{2} /$ d.o.f. $<3$ are taken for further analysis. To reduce the background from the events with a $J / \psi$ produced directly in $p p$ collision, the $t>0.2 \mathrm{ps}$ cut is applied.

To form the $B_{s}^{0} \pi^{ \pm}$candidate, only $B_{s}^{0}$ events with a reconstructed mass in the signal region of $5346.6-5386.6 \mathrm{MeV}$ are included and combined with pion tracks forming a common PV. To maximize the $B_{s}^{0} \pi^{ \pm}$signal to the background ratio, only tracks with transverse momentum $p_{\mathrm{T}}>500 \mathrm{MeV}$ are used. The detailed description of the selection cuts can be found in [6].

\subsection{Fit to data}

The first step was to make the background study in a similar way that the D $\emptyset$ collaboration did and to establish the best background model using real data and MC generated sample. Based 
on a background study, the background model described in [6] was used as default. The signal PDF $F_{s i g}\left(m\left(B_{s}^{0} \pi^{ \pm}\right)\right)$is defined as a convolution of an S-wave Breit-Wigner(BW) distribution with a detector resolution function which is represented by a Gaussian function with a width that is calculated individually for each $B_{s}^{0} \pi^{ \pm}$candidate from the tracking and vertexing error matrices. The signal mass and width are fixed to the central values reported by the $\mathrm{D} \emptyset$ collaboration.

In order to fit the total model, which consists of background and signal PDF, the unbinned maximum-likelihood fit with a per-candidate error was used. The results of the fits are shown in Fig. 1 for two sets of $B_{s}^{0} \pi^{ \pm}$candidates, the first with $p_{\mathrm{T}}>10 \mathrm{GeV}$ and the second with $p_{\mathrm{T}}>15 \mathrm{GeV}$.
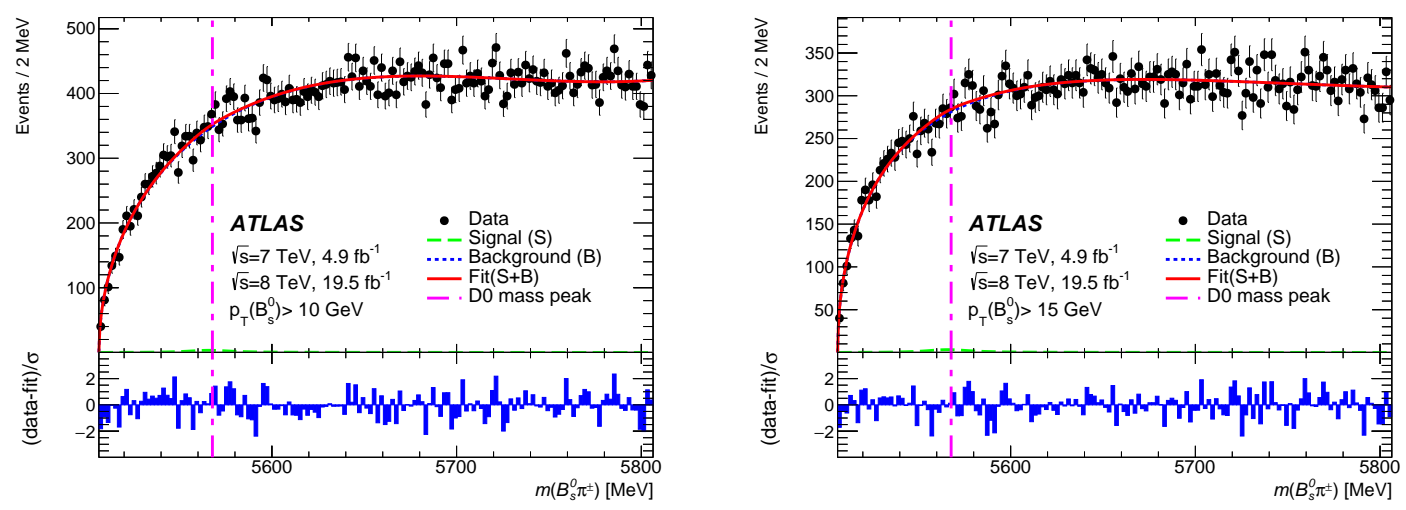

Figure 1: Results of the fit to the $B_{s}^{0} \pi^{ \pm}$mass distribution for candidates with $p_{\mathrm{T}}\left(B_{s}^{0}\right)>10 \mathrm{GeV}$ (left) $p_{\mathrm{T}}\left(B_{s}^{0}\right)>15 \mathrm{GeV}$ and (right). The bottom panels show the difference between each data point and the fit divided by the statistical uncertainty of that point [6].

The extracted values for the number of $B_{s}^{0} \pi^{ \pm}$candidates is $N(X)=60 \pm 140$ for $\left(p_{\mathrm{T}}>10 \mathrm{GeV}\right)$ and $N(X)=-30 \pm 150$ for $\left(p_{\mathrm{T}}>15 \mathrm{GeV}\right)$ and no significant $\mathrm{X}(5568)$ signal was observed. Additional selections such as cuts on the angle between the momenta of the $B_{s}^{0}$ and $\pi^{ \pm}$candidates were investigated and did not produce evidence of a signal.

\subsection{Setting upper limits}

Since no significant signal is observed which is corresponding to the stated properties of the $\mathrm{X}(5568)$ as reported by Ref. [1], upper limits are determined for the number of $B_{s}^{0} \pi^{ \pm}$signal events, $\mathrm{N}(\mathrm{X})$, and for the relative production rate of the $B_{s}^{0} \pi^{ \pm}$and $B_{s}^{0}, \rho_{X}$. The relative production rate within the ATLAS acceptance is defined as follows:

$$
\rho_{X} \equiv \frac{\sigma(p p \rightarrow X+\text { anything }) \times \mathscr{B}\left(X \rightarrow B_{s}^{0} \pi^{ \pm}\right)}{\sigma\left(p p \rightarrow B_{s}^{0}+\text { anything }\right)}=\frac{N(X)}{N\left(B_{s}^{0}\right)} \times \frac{1}{\mathcal{E}^{\text {rel }}(X)},
$$

where the $\sigma$ are the cross-sections related to the produced particles (resonance $\mathrm{X}$ or meson $B_{s}^{0}$ ) within the ATLAS acceptance, $N(X)$ and $N\left(B_{s}^{0}\right)$ are the yields obtained from the fits to invariant mass distributions and $\varepsilon^{\mathrm{rel}}(X)=\frac{\varepsilon(X)}{\varepsilon\left(B_{s}^{0}\right)}$ is a relative efficiency of the $B_{s}^{0} \pi^{ \pm}$state $X$ and the $B_{s}^{0}$ meson. Since $B_{s}$ efficiency cancels, the $\varepsilon^{\text {rel }}$ accounts for the reconstruction and selection efficiencies of the companion pion including the soft pion acceptance.

The upper limit is calculated using the asymptotic approximation from the profile likelihood formalism based on the $\mathrm{CL}_{\mathrm{s}}$ frequentist method. The systematic uncertainties are included in the 
method and systematics effects on $N(X)$ accounts for uncertainties: of D $\emptyset$ measurement of $m_{X}$ and $\Gamma_{X}$; the background model, estimated by replacing the default model by a seventh-order Chebyshev polynomial; the model of the $\mathrm{X}$ resonance, where the default model that assumes $\mathrm{X}$ to have no spin is replaced by a BW P-wave resonance form; and the detector resolution with the default per-candidate mass resolution model replaced by the sum of three Gaussian functions with a common mean. The extracted upper limits at $95 \%$ C.L. are $N(X)<382$ for $p_{\mathrm{T}}\left(B_{s}^{0}\right)>10 \mathrm{GeV}$ and $N(X)<356$ for $p_{\mathrm{T}}\left(B_{s}^{0}\right)>15 \mathrm{GeV}$, respectively.

To extract the upper limits on $\rho_{X}$, the same systematic effects are included with two additional contributions which account for $\varepsilon^{\text {rel }}$ and $N\left(B_{s}^{0}\right)$ uncertainties. These are included into the $\mathrm{CL}_{\mathrm{s}}$ method as additional Gaussian constraints. The resulting upper limits at $95 \%$ C.L. are $\rho_{X}<0.015$ for $p_{\mathrm{T}}\left(B_{s}^{0}\right)>10 \mathrm{GeV}$ and $\rho_{X}<0.016$ for $p_{\mathrm{T}}\left(B_{s}^{0}\right)>15 \mathrm{GeV}$, respectively.

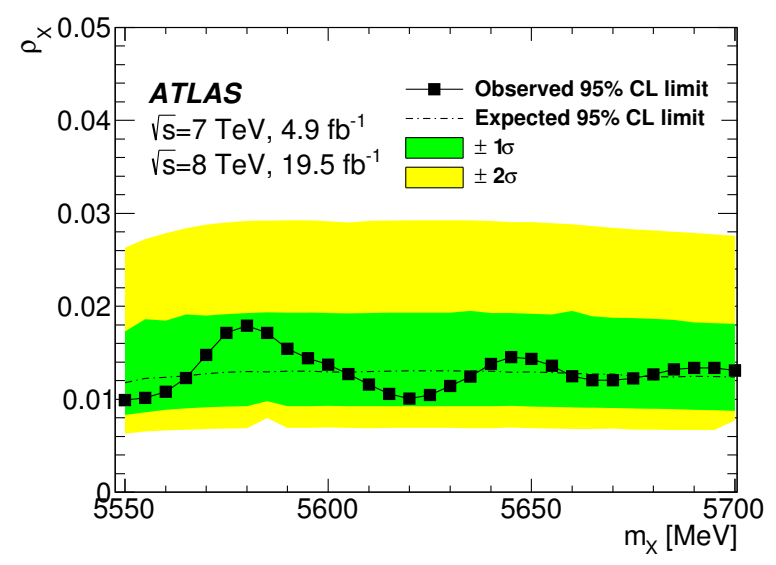

Figure 2: Upper limits on $\rho_{X}$ at $95 \%$ CL (black squares connected by line) at different masses of a hypothetical resonant state $X$ decaying to $B_{s}^{0} \pi^{ \pm}$, for events with $p_{\mathrm{T}}\left(B_{s}^{0}\right)>10 \mathrm{GeV}$. The values include systematic uncertainties. The expected (central black dot-dashed line) $95 \% \mathrm{CL}$ upper limits with $\pm 1 \sigma$ (green) and $\pm 2 \sigma$ (yellow) uncertainty bands on $\rho_{X}$ are shown as functions of the $B_{s}^{0} \pi^{ \pm}$mass [6].

A hypothesis test is performed for the presence of a $B_{s}^{0} \pi^{ \pm}$peak for every $5 \mathrm{MeV}$ step in its mass from 5550 to $5700 \mathrm{MeV}$, with assumption of resonant state described by S-wave BW distribution with BW width of $21.9 \mathrm{MeV}$ and $p_{\mathrm{T}}\left(B_{s}^{0}\right)>10 \mathrm{GeV}$. The mass dependence of resolution and $\varepsilon^{\text {rel }}$ function is included as well as all systematics effects except $\mathrm{X}(5568)$ mass uncertainty. The results are shown in Fig. 2 and are within $\pm 1 \sigma$ of the background only model.

\section{Excited $B_{c}^{ \pm}$Meson}

\subsection{Introduction}

The $B_{c}^{ \pm}$meson was first observed by the CDF experiment in the semileptonic decay mode [8] and since then several searches for its excited state were performed. Since the spectrum and properties of the $B_{c}^{ \pm}$family are predicted by nonrelativistic potential models, perturbative QCD and lattice calculations, any measurement of its excited state would provide tests of the predictions of these models and give useful information on the strong interaction potential. 
The search for excited states of the $B_{c}^{ \pm}$with the ATLAS detector was performed through its hadronic transition to the ground state, with the latter detected in the decay $B_{c}^{ \pm} \rightarrow J / \psi \pi^{ \pm}$[9]. The second S-wave state, $B_{c}^{ \pm}(2 S)$, is predicted to have a mass in the range of $6835-6917 \mathrm{MeV}$ and to have pseudoscalar $\left(0^{-}\right)$and vector $\left(1^{-}\right)$spin states that are predicted to differ in mass by about $20-50 \mathrm{MeV}$.

\subsection{Data and candidate selection}

This study uses $p p$ collision data with $\sqrt{s}=7 \mathrm{TeV}$ collected in 2011 and $\sqrt{s}=8 \mathrm{TeV}$ collected in 2012 with integral luminosity of $4.9 \mathrm{fb}^{-1}$ and $19.2 \mathrm{fb}^{-1}$, respectively. Due to the different centre-of-mass energies and the number of simultaneous $p p$ interactions per bunch crossing, the $B_{c}^{ \pm}$ selection criteria are optimized separately for 7 and $8 \mathrm{TeV}$ data using the corresponding MC samples. The performed analysis is using variable $Q=m\left(B_{c}^{ \pm} \pi \pi\right)-m\left(B_{c}^{ \pm}\right)-2 m\left(\pi^{ \pm}\right)$, where $m\left(B_{c}^{ \pm}\right)$ and $m\left(B_{c}^{ \pm} \pi \pi\right)$ are the offline reconstructed invariant masses of selected candidates and the $m\left(\pi^{ \pm}\right)$is the mass of charged pion. This technique helps the analysis significantly by improving resolution.

The first step in the analysis is the $J / \psi$ candidate selection, where the muon tracks are fit to a common vertex where the $p_{\mathrm{T}}$ of higher- $p_{\mathrm{T}}$ muon candidate is required to be above $6 \mathrm{GeV}$ and $p_{\mathrm{T}}$ of lower- $p_{\mathrm{T}}$ muon candidate is required to be above $4 \mathrm{GeV}$. Only di-muon candidates in range of $\pm 3 \sigma$ around the world average $J / \psi$ mass are then used for the analysis.

The $B_{c}^{ \pm}$selection was optimized using MC events to maximize the $S / \sqrt{S+B}$ ratio. The $B_{c}^{ \pm}$ candidates are reconstructed by fitting two muon tracks from the $J / \psi$ candidate together with a pion candidate track to a common vertex, where the $p_{\mathrm{T}}$ of pion candidate is required to be larger than $4 \mathrm{GeV}$. The vertex fit of $J / \psi \pi$ must have $\chi^{2} /$ d.o.f. $<2$ for $7 \mathrm{TeV}$ and $\chi^{2} /$ d.o.f. $<1.5$ for $8 \mathrm{TeV}$. The $p_{\mathrm{T}}$ of the $B_{c}^{ \pm}$must be larger than 15 and $18 \mathrm{GeV}$ for 7 and $8 \mathrm{GeV}$, respectively.

The invariant mass distribution of $B_{c}^{ \pm}$candidates is fitted using unbinned maximum likelihood fit, with a Gaussian function modeling the signal and an exponential function modeling the background shape, where the fit result is shown in Fig. 3.
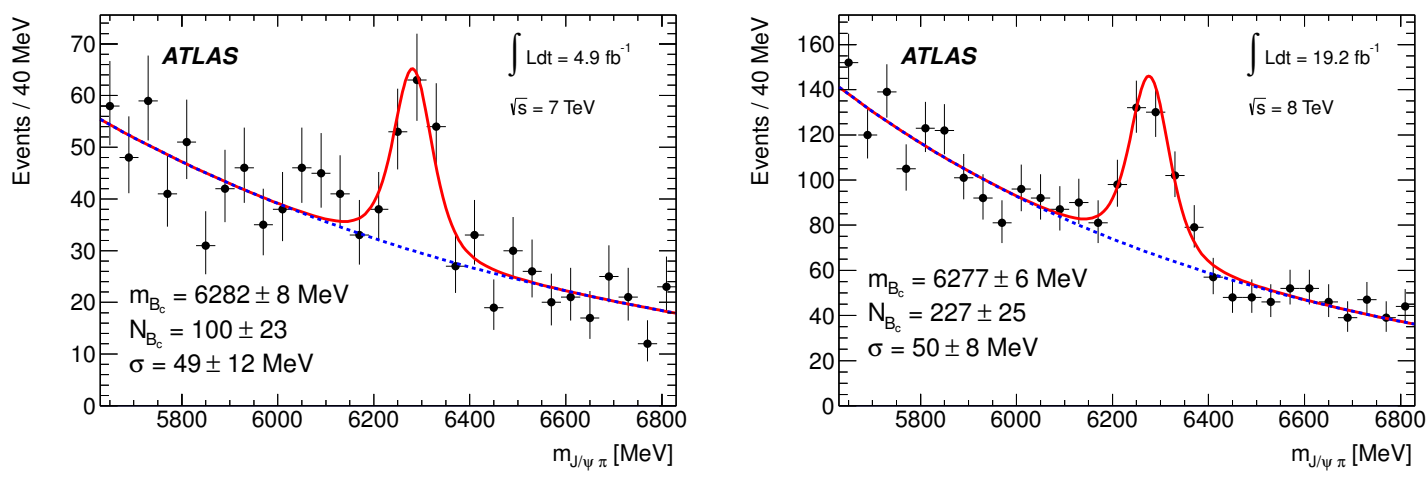

Figure 3: Invariant mass distributions of the reconstructed $B_{c}^{ \pm} \rightarrow J / \psi \pi$ candidates in $7 \mathrm{TeV}$ data (left) and in $8 \mathrm{TeV}$ data (right). The data are represented by the points with error bars (statistical only). The solid line is the projection of the results of the unbinned maximum likelihood fit to all candidates in the mass range $5620-6820 \mathrm{MeV}$. The dashed line is the projection of the background component of the same fit [9].

The candidates within $\pm 3 \sigma$ of the fitted $B_{c}^{ \pm}$mass are then used for further analysis and are combined with two-pion candidate tracks associated with the corresponding primary vertex. The 
$p_{\mathrm{T}}$ of each pion candidate must be larger than $400 \mathrm{MeV}$. All selected tracks are then refitted simultaneously with decay topology constraints and only the candidate with best $\chi^{2}$ is considered in each event. Detailed description of the selection can be found in Ref. [9].

\section{$2.3 B_{c}^{ \pm}(2 S)$ Fit}

For the selected candidates, the mass difference distribution was fit using maximum likelihood fit and right-charge combinations. The wrong-charge combination were kept for the comparison with the combinatorial background shape in the right-charge combinations. The signal shape is modelled by a Gaussian function and for the background the third-order polynomial was used. The background shape resulting from the fit is verified to be consistent with the wrong-charge combinations (which are not used to constrain the model in the right-charge fit). The resulting mass difference distribution is shown in Fig. 4. The extracted parameters with statistical uncertainty only are $Q=288.2 \pm 5.1 \mathrm{MeV}$, yield $22 \pm 6$ and Gaussian width $18.2 \pm 3.8 \mathrm{MeV}$ for the $7 \mathrm{TeV}$ data and $Q=288.4 \pm 4.8 \mathrm{MeV}$, yield $35 \pm 13$ and Gaussian width $17.0 \pm 4.0 \mathrm{MeV}$ for the $8 \mathrm{TeV}$ data.
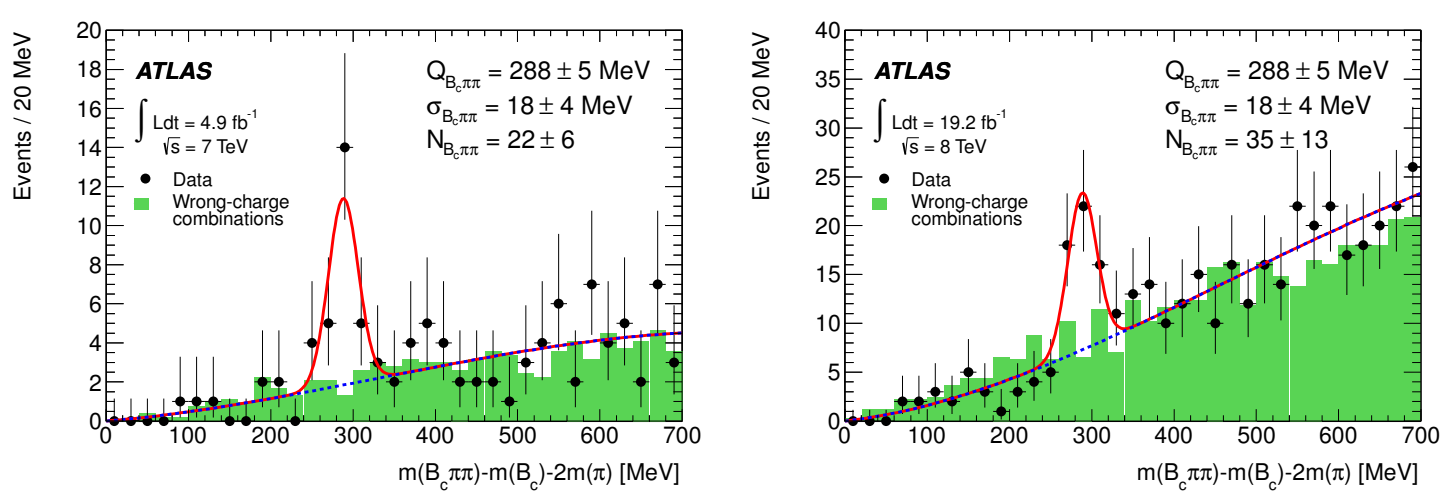

Figure 4: The distribution for the right-charge combinations (points with error bars) and for the same (wrong) pion charge combinations (shaded histogram) in $7 \mathrm{TeV}$ data (left) and in $8 \mathrm{TeV}$ data (right). The wrong-charge combinations are normalized to the same yield as the right-charge background. The solid line is the projection of the results of the unbinned maximum likelihood fit to all candidates in the range $0-700 \mathrm{MeV}$. The dashed line is the projection of the background component of the same fit [9].

For the systematic uncertainties, alternative background and signal functions were used. A BW contribution was tested in convolution with the Gaussian function and the impact was found to be negligible, implying that the natural width of the structure is small relative to the detector resolution. The uncertainty on the position of the peak is dominated by the fitting procedure and is estimated to be about 3.6 MeV. Other systematic effects accounts for the effects of the mass bias in the selection of the candidate with the best $\chi^{2}$ of the vertex, uncertainty on the pion momentum scale and the residual uncertainty from the $B_{c}^{ \pm}$candidate mass in the mass difference distribution. The total averaged systematic uncertainty propagated to the mass value of the new resonance is approximately $4.1 \mathrm{MeV}$.

\subsection{Significance}

In order to establish the significance of the observation, pseudoexperiments with large number of background only mass difference distributions are generated. Parameters of the generation are 
taken from the fit with their uncertainties to account for systematic effects. The mean invariant mass value of the signal contribution is left free to vary within the theoretical predicted range $(6835-6917 \mathrm{MeV})$ to evaluate the "look-elsewhere effect". Measured significance is $3.7 \sigma$ for the $7 \mathrm{TeV}$ data and $4.5 \sigma$ for the $8 \mathrm{TeV}$ data. The significance of the combined 7 and $8 \mathrm{TeV}$ dataset is $5.2 \sigma$. The local significance of the observation, obtained by fixing the mean value of the signal component, is $5.4 \sigma$.

\section{Conclusion}

A search for a new state $X(5568)$ decaying to $B_{s}^{0} \pi^{ \pm}$, as reported by the $\mathrm{D} \emptyset$ collaboration, was performed by ATLAS, using $4.9 \mathrm{fb}^{-1}$ of $p p$ collision data at $7 \mathrm{TeV}$ and $19.5 \mathrm{fb}^{-1}$ at $8 \mathrm{TeV}$. No significant signal was found, within the analysis acceptance. The upper limits on the number of signal events $N(X)$ and on its production rate relative to $B_{s}^{0}$ mesons were measured. The published upper limits at $95 \%$ C.L. are $N(X)<382$ and $\rho_{X}<0.015$ for $p_{\mathrm{T}}\left(B_{s}^{0}\right)>10 \mathrm{GeV}$ and $N(X)<356$ and $\rho_{X}<0.016$ for $p_{\mathrm{T}}\left(B_{s}^{0}\right)>15 \mathrm{GeV}$. The hypothesis test for $\mathrm{X}(5568)$ state was performed and across the full range is consistent with background only model.

A search for an excited state of the $B_{c}^{ \pm}$resonance was performed in $B_{c}^{ \pm}(2 S) \rightarrow B_{c}^{ \pm}(1 S) \pi^{ \pm} \pi^{\mp}$ channel using $p p$ collision data with $4.9 \mathrm{fb}^{-1}$ at $7 \mathrm{TeV}$ and $19.5 \mathrm{fb}^{-1}$ at $8 \mathrm{TeV}$ at the ATLAS detector. A new resonant state is observed at a mass difference of $Q=288.3 \pm 3.5$ (stat.) \pm 4.1 (syst.) $\mathrm{MeV}$ corresponding to an invariant mass of $6842 \pm 4$ (stat.) \pm 5 (syst.) MeV. The significance of the observation is $5.2 \sigma$ with the look elsewhere effect taken into account, and the local significance is $5.4 \sigma$. The mass of observed structure is consistent with the predicted mass of the $B_{c}^{ \pm}(2 S)$ with no $B_{c}^{*}(2 S)$ hypothesis.

\section{References}

[1] V. M. Abazov et al., "Evidence for a $B_{s}^{0} \pi^{ \pm}$state," Phys. Rev. Lett., vol. 117, no. 2, p. 022003, 2016.

[2] R. Aaij et al., "Search for Structure in the $B_{s}^{0} \pi^{ \pm}$Invariant Mass Spectrum," Phys. Rev. Lett., vol. 117, no. 15, p. 152003, 2016. [Addendum: Phys. Rev. Lett.118,no.10,109904(2017)].

[3] A. M. Sirunyan et al., "Search for the $\mathrm{X}(5568)$ state decaying into $\mathrm{B}_{\mathrm{s}}^{0} \pi^{ \pm}$in proton-proton collisions at $\sqrt{s}=8$ TeV," Phys. Rev. Lett., vol. 120, no. 20, p. 202005, 2018.

[4] T. Aaltonen et al., "A search for the exotic meson $X(5568)$ with the Collider Detector at Fermilab," Phys. Rev. Lett., vol. 120, no. 20, p. 202006, 2018.

[5] V. M. Abazov et al., "Study of the $X^{ \pm}(5568)$ state with semileptonic decays of the $B_{s}^{0}$ meson," Phys. Rev., vol. D97, no. 9, p. 092004, 2018.

[6] ATLAS Collaboration, "Search for a Structure in the $B_{s}^{0} \pi^{ \pm}$Invariant Mass Spectrum with the ATLAS Experiment," Phys. Rev. Lett., vol. 120, no. 20, p. 202007, 2018.

[7] ATLAS Collaboration, "The ATLAS Experiment at the CERN Large Hadron Collider," JINST, vol. 3, p. S08003, 2008.

[8] F. Abe et al., "Observation of the $B_{c}$ meson in $p \bar{p}$ collisions at $\sqrt{s}=1.8 \mathrm{TeV}$," Phys. Rev. Lett., vol. 81 , pp. 2432-2437, 1998.

[9] ATLAS Collaboration, "Observation of an Excited $B_{c}^{ \pm}$Meson State with the ATLAS Detector," Phys. Rev. Lett., vol. 113, no. 21, p. 212004, 2014. 